\title{
Framework for analyzing hyper-viscoelastic polymers
}

Cite as: AIP Conference Proceedings 1979, 090013 (2018); https://doi.org/10.1063/1.5044870 Published Online: 03 July 2018

Akash Trivedi, and Clive Siviour

\section{ARTICLES YOU MAY BE INTERESTED IN}

High strain rate characterization of polymers

AIP Conference Proceedings 1793, 060029 (2017); https://doi.org/10.1063/1.4971585

A split Hopkinson pressure bar technique for measuring the stress relaxation behavior of polymers on microsecond timescales

AIP Conference Proceedings 1979, 090015 (2018); https://doi.org/10.1063/1.5044872

Fragment penetrating injury to long bones

AIP Conference Proceedings 1979, 090011 (2018); https://doi.org/10.1063/1.5044868
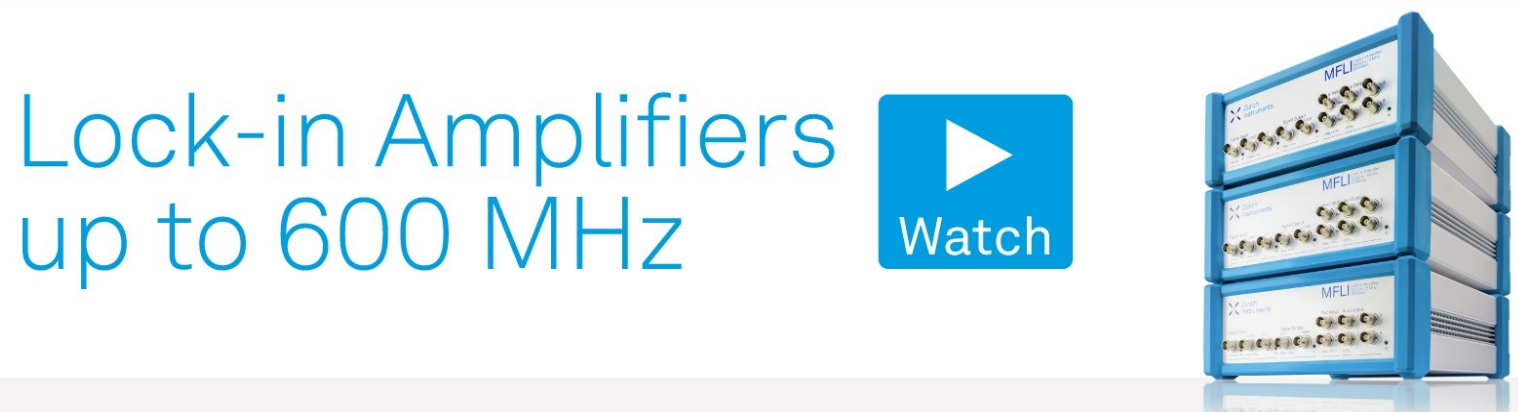


\title{
Framework for Analyzing Hyper-Viscoelastic Polymers
}

\author{
Akash Trivedi $^{1, a)}$ and Clive Siviour ${ }^{1}$ \\ ${ }^{1}$ Solid Mechanics and Materials Engineering Group, Department of Engineering Science, \\ Parks Road, Oxford, OX1 3PJ, United Kingdom \\ a)Corresponding author: akash.trivedi@eng.ox.ac.uk
}

\begin{abstract}
Hyper-viscoelastic polymers have multiple areas of application including aerospace, biomedicine, and automotive. Understanding their mechanical responses is therefore extremely important, particularly because they often exhibit strong rate and temperature dependence, including a low temperature brittle transition. Relationships between the response at various strain rates and temperatures are investigated and a framework developed to predict response at rates where experiments are unfeasible. A mas-ter curve showing the rate dependences of the storage and loss moduli at a reference temperature is constructed using the results of a dynamic mechanical analysis (DMA) experiment. A frequency sweep spanning two decades and a temperature range from pre-glass transition to pre-melt is used. A fractional derivative model is fitted to the experimental data, and this models parameters are used to show how the stress-strain relationships at a desired strain rate could be derived.
\end{abstract}

\section{INTRODUCTION}

The overarching aim of this research is to be able to predict the mechanical response of polymers, their composites, and similar types of materials at high strain rates purely by knowing their composition, and by performing the minimal set of experimental tests. In the current paper, an indirect method for obtaining the high strain rate properties is explored and a framework developed that would allow future characterisation of similar materials.

One may assume the easiest method of obtaining the high strain rate properties of polymers would be to test them at the rate desired. This sounds ideal, but is in fact challenging when it comes to soft polymers. Due to the low stiffness of the material, the wavespeed is also low, and therefore stress equilibrium is not easily reached. Under dynamic loading, the measured forces at the end of the specimen may not be representative of the material response but instead a combination of the material and specimen behaviour. Furthermore, in the standard split-Hopkinson pressure bar (SHPB), the forces supported by softer specimens are low and so measurements are difficult.This can be improved by matching the bar and specimen impedances [1,2].

In previous research [3, 4], it has been demonstrated that a qualitative understanding of the ratetemperature interdependence may be found through a one-to-one equivalence between a characteristic stress at a high strain rate and correlating it to the same characteristic stress at a lower strain rate, but also a lower temperature. Although this method allows for an appreciation of the stress at a particular level of strain for a high strain rate experiment using results from lower strain rate tests, the full stress-strain relationship cannot be obtained in this manner and neither does it allow for a prediction of the mechanical response.

Based on the classic result of the time-temperature superposition (TTS) principle, one can relate changes in rate to changes in temperature [5,6,7]. Master curves and shift factors detailing the material's rate and temperature dependences can be obtained and then a suitable constitutive model could be used for predicting the full stress-strain response over a range of strain rates.

Shock Compression of Condensed Matter - 2017

AIP Conf. Proc. 1979, 090013-1-090013-5; https://doi.org/10.1063/1.5044870

Published by AIP Publishing. 978-0-7354-1693-2/\$30.00

090013-1 


\section{EXPERIMENTAL OVERVIEW}

The polymeric material that was used in all experiments was a commercial black neoprene rubber supplied by Brammer, UK in the form of a $5 \mathrm{~mm}$ sheet. For the DMA experiments, rectangular samples of the material were cut with dimensions $50 \times 10 \times 5 \mathrm{~mm}$. The experiments were conducted on a TA Instruments Q800 and all experiments were performed in the dual cantilever configuration. Full calibration of the apparatus was performed in occardance with the protocol set out by TA prior to data acquisition. An isothermal frequency sweep in $2{ }^{\circ} \mathrm{C}$ increments was used with values of $0.5,2,5$, and $10 \mathrm{~Hz}$ and a temperature range from $-50^{\circ} \mathrm{C}$ to $80^{\circ} \mathrm{C}$.

For the compression experiments, cylindrical samples with a diameter of $5 \mathrm{~mm}$ were cut out from the sheet. These experiments were conducted at a variety of strain rates from $10^{-3}-10^{3} \mathrm{~s}^{-1}$ at a temperature of $25^{\circ} \mathrm{C}$, and at a variety of temperatures from $-100^{\circ} \mathrm{C}$ to $80^{\circ} \mathrm{C}$ at a rate of $10^{-2} \mathrm{~s}^{-1}$.

A commercially available Instron 5980 electromechanical static testing machine was used for experiments at low strain rates $\left(10^{-3} \mathrm{~s}^{-1}-10^{-1} \mathrm{~s}^{-1}\right)$. For low load cases (typically temperatures above the glass transistion temperature $\left(T_{g}\right)$ of the polymer), the machine cross-head displacement was used to obtain strain and a standard Instron $5 \mathrm{kN}$ load cell for force measurements. Preliminary experiments showed that at temperatures above $T_{g}$, there was minimal machine compliance, so the cross-head displacement gave an accurate measure of the movement of the loading platens. Constant true strain rate control was used in all experiments.

An environmental chamber with a liquid nitrogen feed was used for testing at sub-ambient temperatures. Temperature was controlled with the default thermostat which was accurate to $\pm 1^{\circ} \mathrm{C}$. Since the environmental chambers feedback control thermocouple is situated at the back of the chamber away from the specimen, a second thermocouple was inserted into the loading platen itself to measure the temperature as close to the specimen as possible. In order to verify thermal equilibrium, only when this thermocouple reading matched the chamber thermocouple to within $\pm 0.5^{\circ} \mathrm{C}$, was the test conducted. The same chamber and temperature measurements were used for supra-ambient temperature experiments. Temperatures were recorded with a National Instruments USB thermocouple reader. In all cases, thermal expansion of the load rig was corrected by ensuring zero load at the start of each test.

Due to higher loads experienced at lower temperatures (typically below $T_{g}$ ), there was a need to increase the load cell capacity to $50 \mathrm{kN}$. Also, as the higher loads could lead to rig compliance effects, an extensometer was used to obtain local measurements of the platen displacement.

For the compressive room temperature experiments at medium rate $\left(1 \mathrm{~s}^{-1}-10^{2} \mathrm{~s}^{-1}\right)$, a custom-built hydraulic load frame was used. Using linear variable differential transformers (LVDT) it was possible to measure the displacement of the anvils, and the force was measured using a strain gauge based load cell. This then allowed the stress-strain relationship to be determined.

For compression experiments at a strain rate of the order $10^{3} \mathrm{~s}^{-1}$, an in-house SHPB was used. Full details of the analysis procedure of using a SHPB for the testing of soft polymers can be found in [8]. In this research, Ti - 6Al - 4V alloy bars with a $12.7 \mathrm{~mm}$ diameter were used. Using compressed gas to fire the striker bar, speeds of up to around $20 \mathrm{~m} \mathrm{~s}^{-1}$ were achievable giving the possibility of testing at rates of order $10^{3} \mathrm{~s}^{-1}$.

\section{RESULTS AND ANALYSIS}

\section{Rate-temperature equivalence}

A compilation of stress-strain curves for room temperature (RT) experiments at varying rates can be found in Figure 1(a). Qualitatively, there is a hyperelastic behaviour dominance at large strain and with increasing rate, a larger low strain viscoelastic behaviour. The results of the compression experiments conducted at a variety of temperatures and a strain rate of $10^{-2} \mathrm{~s}^{-1}$ are presented in Figure 1(b). A simple observation is that the lower the temperature (in particular temperatures below the $T_{g}$ ), the stiffer, stronger, and more brittle the polymer. At higher temperatures (above the $T_{g}$ ), the polymer behaves as a hyperelastic rubber and eventually relaxes back to the original shape once the load is removed. It is key to observe that both results of the varying rate experiments and the varying temperature experiments exhibit some variant of an increasing trend in stress. This forms the basis of the rate-temperature equivalence.

It is possible to map out an equivalence between rate and temperature as shown in Figure 1(c). Since increasing rate leads to a higher stress, as does a decreasing temperature, by reversing the temperature axis, the similarities between these dependencies are clearer. For example, if a qualitative understanding of the characteristic stress reponse at a rate of $10^{3} \mathrm{~s}^{-1}$ was desired, then by using Figure 1(c) for reference, a quasi-static test at a rate of $10^{-2} \mathrm{~s}^{-1}$ and a temperature of around $-65^{\circ} \mathrm{C}$ could be performed. 
(a)

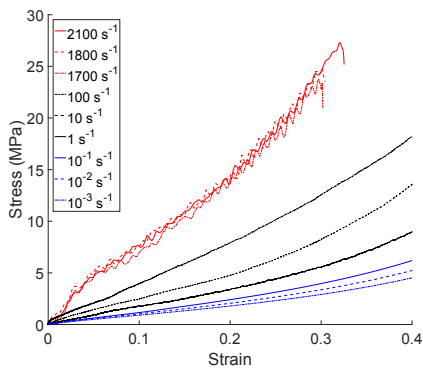

(b)

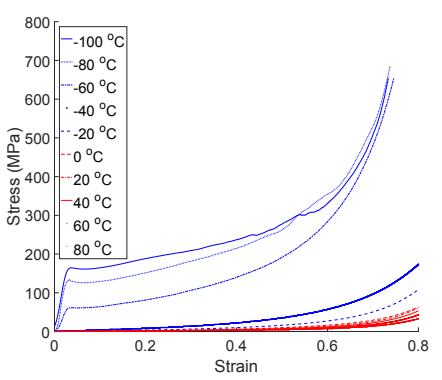

(c)

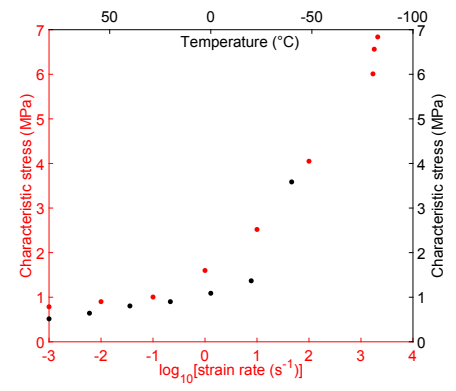

FIGURE 1. Results of the (a) varying rate experiments, (b) varying temperature experiments, and (c) rate-temperature equivalence.

\section{DMA experiments}

It is clear that both temperature and frequency both affect the stiffness of the specimens. As temperature increases, the modulus decreases as the polymer goes through its $T_{g}$ and becomes rubbery. A lower frequency also shows lower moduli values, similar to the effects at higher temperature. As was described in the previous section, this forms the basis of the classic result of TTS where high frequency results are analagous to low temperature results, and vice versa.

Figure 2(a) shows the shift factors required in order to translate each isotherm horizontally to overlap with the adjacent one. Doing this for each isotherm excluding the one for the reference RT value of $25^{\circ} \mathrm{C}$, the master curves for the storage and loss moduli can be obtained as shown in Figure 2(b).

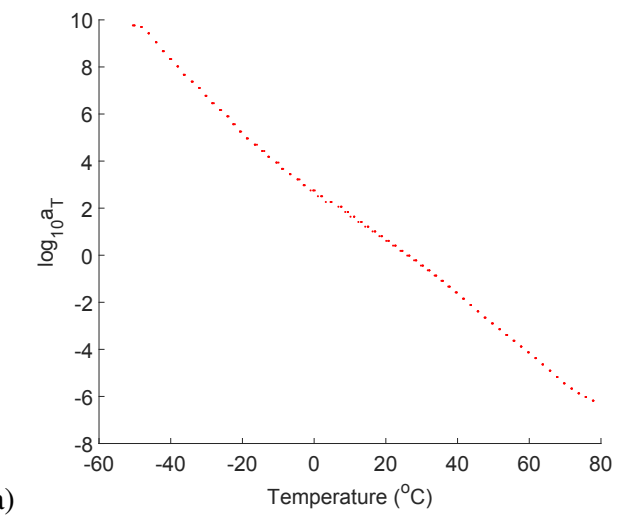

(b)

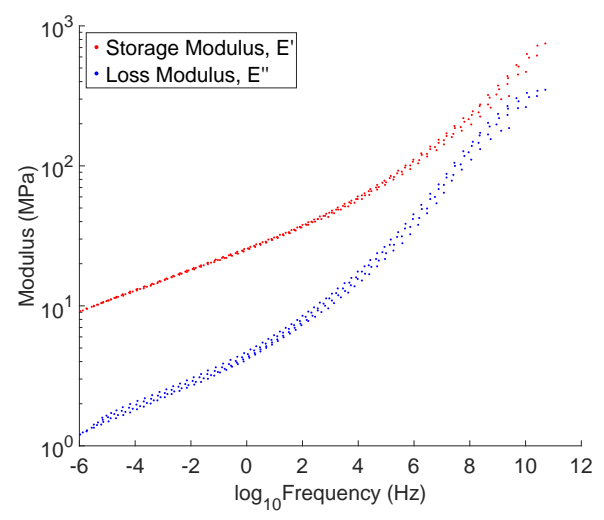

FIGURE 2. Results from DMA experiments (a) shift factors as a function of temperature and (b) storage and loss moduli master curves.

\section{CONSTITUTIVE MODELLING}

A basic hyperelastic model for rubber would miss the low strain viscoelasticity at the highest strain rate experiments, and so a hyper-viscoelastic (HV) model was developed where the overall stress behaviour was decomposed into the rate-independent hyperelastic component obtained from the lowest rate quasi-static experiment and the rate-dependent viscoelastic component. This is shown diagrammatically in Figure 3(a). The viscoelastic model that was selected for this current research was one of the most commonly used ones; the standard linear solid (SLS) model (shown in Figure $3(\mathrm{~b}))$.

The hyperelastic stress component, $\sigma_{h}$, is defined by the one-term Ogden fit to the quasi-static test at $10^{-3} \mathrm{~s}^{-1}$ with the values of $\alpha=2.32$ and $\mu=-2.00$.

$$
\sigma_{h}=\frac{2 \mu}{\alpha}\left[\lambda^{\alpha-1}-\lambda^{-\left(1+\frac{\alpha}{2}\right)}\right]
$$


The viscoelastic stress component, $\sigma_{v}$, can be found using the following equation for a particular value of the strain rate.

$$
\sigma_{v}=\frac{E_{1} \dot{\varepsilon}}{E_{1}+E_{2}}\left[E_{2} t+E_{1} \tau\left(1-e^{-\frac{t}{\tau}}\right)\right]
$$

In fitting to the $1.3 \mathrm{~s}^{-1}$ data: $E_{1}=19 \mathrm{MPa}, E_{2}=8.5 \mathrm{MPa}$, and $\tau=2.8 \times 10^{-2} \mathrm{~s}$. For the $2100 \mathrm{~s}^{-1} \mathrm{data}$ $E_{1}=130 \mathrm{MPa}, E_{2}=67 \mathrm{MPa}$, and $\tau=9.3 \times 10^{-6} \mathrm{~s}$. The HV fits can be seen in Figure 4(a) for $1.3 \mathrm{~s}^{-1}$ and Figure 4(b) for $2100 \mathrm{~s}^{-1}$. However, as good as the HV model fits are to the experimental data, this is not the ideal case since the SLS moduli parameters should remain constant and not vary with rate. Rate dependence has already been shown to be included in the results of the DMA test and for this reason a model based on a fit to the DMA results is sought.

(a)

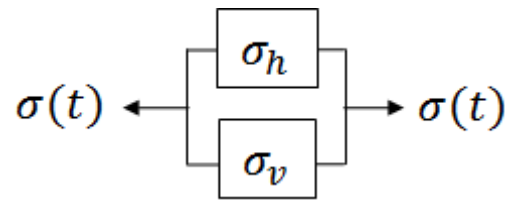

(b)

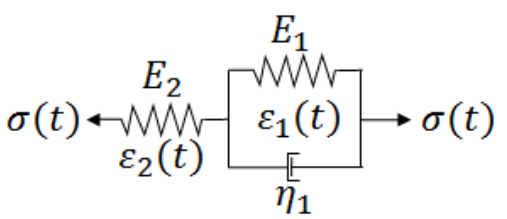

FIGURE 3. Diagrams of the constitutive models used: (a) hyper-viscoelastic (b) SLS.

(a)

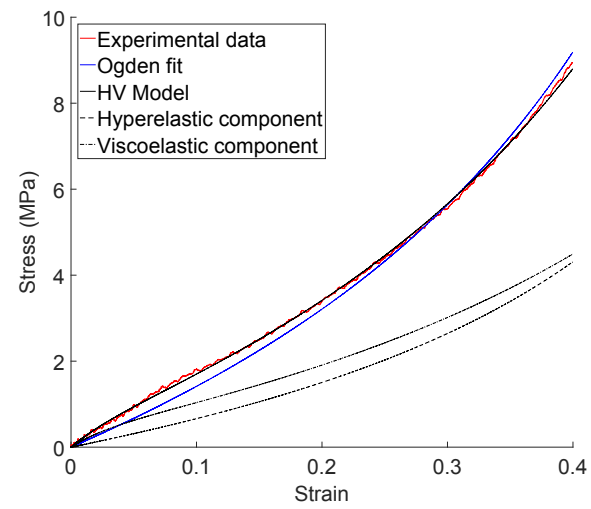

(b)

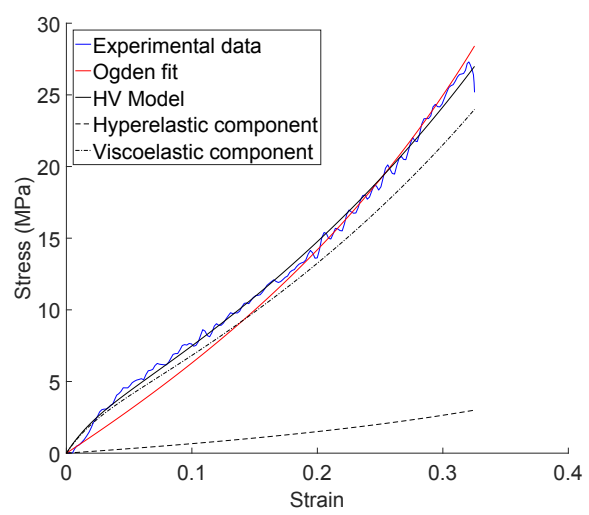

FIGURE 4. Hyper-viscoelastic model fits to experiments conducted at RT for (a) $\dot{\varepsilon}=1.3 \mathrm{~s}^{-1}$ and (b) $\dot{\varepsilon}=2100 \mathrm{~s}^{-1}$.

A second method to describe the viscoelastic behaviour is to use a fractional derivative (FD) model. The fourterm FD model is shown diagrammatically in Figure 5(a). The DMA results were shown in Figure 2(b) and using this four-term FD model, a fit to the DMA test over great spans of rates can also be produced as shown in Figure 5(b). The storage and loss moduli are obtained using the following equation.

$$
E=E^{\prime}+i E^{\prime \prime}=\left[E_{0}+E_{1}\left(\frac{1}{1+(i \omega \tau)^{-\beta}}\right)\right]
$$

Currently, parameters can be selected based on a fit to the storage modulus or the loss modulus. It has not been yet investigated whether or not a simultaneous solution exists to provide a common set of parameters for best fitting to both curves. In fitting to the storage modulus: $E_{0}=7.6 \mathrm{MPa}, E_{1}=0.27 \mathrm{MPa}, \tau=1.4 \times 10^{-8} \mathrm{~s}$, and $\beta=0.13$. In fitting to the loss modulus: $E_{0}=0.45 \mathrm{MPa}, E_{1}=450 \mathrm{MPa}, \tau=3.9 \times 10^{-9} \mathrm{~s}$, and $\beta=0.17$.

\section{CONCLUSIONS}

This paper presents current research on providing a framework for better understanding mechanical properties of polymers at high strain rates. This has been done by fitting a hyper-viscoelastic model to intermediate and high strain 
(a)

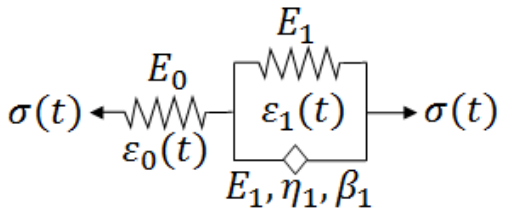

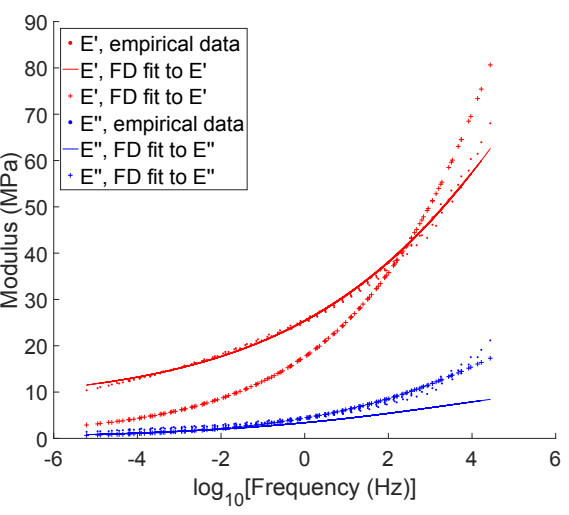

FIGURE 5. (a) Diagrammatic representation of a 4-term FD model and (b) FD model optimally fit to master curves from DMA results truncated for experimentally relevant frequencies.

rate compression results and a fractional derivative model to DMA data. This provides evidence to support a concept where the high strain rate response of the polymer may be obtained from a constitutive model based on only quasistatic and DMA experiments.

Going forward, it is intended to use the rate dependent behaviour captured by the FD model fits to the DMA results and incorporate it with a hyperelastic component to fully describe the stress-strain response of the polymer at any appropriate strain rate. The predictive intention of the framework also needs to be verified with the investigation of previously unexplored strain rates; finding the stress-strain relationship from the model before doing the actual experiment to see whether it is indeed correct as expected. An FEA model will also be produced which will be able to simulate the experimental behaviour and can be used to verify the predictive framework.

Overcoming these obstacles will allow for a truly cohesive framework to be developed to allow for the prediction of high strain rate properties of hyper-viscoelastic polymers and their composites.

\section{ACKNOWLEDGMENTS}

This material is based upon work supported by the Air Force Office of Scientific Research, Air Force Material Command, USAF under Award No. FA9550-15-1-0448. Any opinions, findings, and conclusions or recommendations expressed in this publication are those of the author(s) and do not necessarily reflect the views of the Air Force Office of Scientific Research, Air Force Materiel Command, USAF.

\section{REFERENCES}

[1] W. Chen, B. Zhang, and M. J. Forrestal, Experimental Mechanics 39, 81-85 (1999).

[2] C. Siviour, P. Laity, W. Proud, J. Field, D. Porter, P. Church, P. Gould, and W. Huntingdon-Thresher, Proceedings of the Royal Society A: Mathematical, Physical and Engineering Sciences 464, 1229-1255 (2008).

[3] C. Siviour, S. Walley, W. Proud, and J. Field, Polymer 46, 546-555 (2005).

[4] C. R. Siviour, AIP Conference Proceedings 1793, p. 060029 (2017).

[5] C. Roland, p. 332 (2011).

[6] M. J. Kendall and C. R. Siviour, Proceedings of the Royal Society A: Mathematical, Physical and Engineering Sciences 470 (2014).

[7] C. R. Siviour and J. L. Jordan, Journal of Dynamic Behavior of Materials 2, 15-32 (2016).

[8] G. T. Gray III and W. R. Blumenthal, Split-Hopkinson Pressure Bar Testing of Soft Materials, 2000. 